\title{
Estudo Termoquímico na Produção de Aditivo de Gasolina Tert-Amyl-Methyl-Ether (TAME)
}

\section{Thermochemical Study of Gasoline Additive Production Tert-Amyl-Methyl-Ether (TAME)}

\section{Estudio Termoquímico en la Producción de Aditivo de Gasolina Tert-Amyl-Methyl-Ether (TAME)}

\author{
Jeffrey Leon-Pulido*; Ivan D. Santiago-Cespedes; Angel Dario Gonzalez-Delgado²; \\ Javier Becerra-Navas; ; Viktor Oswaldo Cárdenas-Concha ${ }^{4}$; José Roberto Nunhez \\ ${ }^{1}$ Departamento de Procesos, Universidad EAN, Bogota-Colombia* \\ ${ }^{2}$ Departamento de Ingeniería Química, Universidad de Cartagena, Colombia \\ ${ }^{3}$ Escuela de Ingeniería Química, Universidad Industrial de Santander, Bucaramanga-Colombia \\ ${ }^{4}$ Laboratório de Biotecnologia e Sistemas Naturais, Universidade Federal de São Paulo, SP-Brasil \\ ${ }^{5}$ Faculdade de Engenharia Química, Universidade Estadual de Campinas, SP-Brasil \\ *jleonp@universidadean.edu.co
}

Fecha recepción: 28 de enero de 2019 Fecha aceptación: Junio 04 de 2019

\begin{abstract}
Resumen
Los procesos de producción reactivos y de separación son ampliamente usados en la industria de procesos en general. Específicamente, el proceso de destilación necesita del estudio del equilibrio de las mezclas siendo fundamental para el proceso de separación. En el caso del Tert-Amyl-MethylEther (TAME) funciona como aditivo oxigenador, mejorando el rendimiento mecánico del vehículo. De este modo, el estudio del comportamiento termoquímico y de las fenomenologías de los diferentes componentes involucrados en el sistema de producción del TAME son calculadas para el posterior entendimiento del proyecto de purificación. Siendo así, el análisis de los componentes mezclados haciendo uso del simulador de procesos Aspen Plus $V 9 \circledast$ el objetivo de estudio. Para ello el modelo termodinámico UNIFAC fué utilizado para estimar los parámetros binarios del equilibrio de los reactivos y reactantes calculados con el simulador. La observación de los aspectos analizados presenta la presencia de azeótropos en diferentes condiciones de temperatura, nueve puntos para diferentes temperaturas de mezclas binarias fueron estimados en el estudio termoquímico.
\end{abstract}

Palabras clave: TAME; Termoquímica; Equilibrio; Simulación; Aditivo de Gasolina.

\begin{abstract}
Reactive production and separation processes are widely used in the process industry in general. Specifically, the distillation process requires the equilibrium study of the mixtures are fundamental to the separation process. In the case of Tert-Amyl-Methyl-Ether (TAME) works as an oxygenator additive, improving the mechanical performance of the vehicle. Thus, the study of the thermochemical behavior and phenomena of the different components involved in the TAME production system are calculated for the later understanding of the purification project. Therefore, the objective of the study is to analyze the mixed components using the Aspen Plus $\mathrm{V} 9 \AA$ process simulator. The UNIFAC thermodynamic model was used to estimate the equilibrium binary parameters of the reagents and reactives calculated with the simulator. The observation of the analyzed aspects presents the presence of azeotrops in different
\end{abstract}

Cita: Leon-Pulido J, Santiago Cespedes ID, Gonaez-Delgado Angel Dario, Becerra Navas J, Cárdenas Concha VO, Nunhez JR. Estudo Termoquímico na Produção de Aditivo de Gasolina Tert-Amyl-Methyl-Ether (TAME). rev.ion. 2019;32(1):87-95. doi:10.18273/revion.v32n12019008 
rev.ion. 2019;32(1):87-95. Bucaramanga (Colombia).

temperature conditions, nine points for different temperatures of binary mixtures were estimated in the thermochemical study.

Keywords: TAME; Thermochemical; Equilibrium; Simulation; Additive of Petrol.

\section{Resumo}

Os processos de produção reativos e de separação são amplamente usados na indústria química em geral. A destilação precisa do estudo do equilibro das misturas pois é fundamental no processo de separação. No caso do Tert-Amyl-Methyl-Ether (TAME) funciona como aditivo oxigenador, melhorando o rendimento mecânico. Desse modo, o estudo do comportamento termoquímico e fenomenológico dos diferentes componentes, envolvidos no sistema de produção do TAME foram definidos para posterior entendimento do projeto de purificação. Sendo assim, o objetivo deste estudo é a análise dos componentes na mistura fazendo uso do simulador de processos Aspen Plus $V 9 \circledast$. Para isto o modelo termodinâmico UNIFAC foi utilizado para estimar os parâmetros binários de equilíbrio dos reagentes e reativos calculados com o simulador. Decorrente da análise realizada, azeótropos são apresentados em diferentes condições de temperatura. Nove pontos para diferentes temperaturas de misturas binarias foram estimados no estudo termoquímico.

Palavras chaves: TAME; Termoquímica; Equilíbrio; Simulação; Aditivo de Gasolina.

\section{Introdução}

Os estudos termoquímicos foram desenvolvidos com o intuito de estudar o processo de projeto conceitual do sistema de separação e reação do Tert-Amyl-Methyl-Ether (TAME). O sistema procura a implementação dos processos de separação e reação em uma unidade de Destilação reativa - RD, comportamentos a diferentes pressões e os perfis de temperaturas consequentes. Sabe-se que na produção do TAME (Tert-amyl-methyl-ether) se descreve uma reação exotérmica que acontece em presença do metanol [1]. Atualmente, as indústrias procuram alternativas de melhoramento de processos e aproveitamento de recursos, assim, a destilação reativa é uma alternativa onde são combinadas a reação química e a separação em um único equipamento. $\mathrm{Na}$ síntese do TAME a destilação reativa apresenta uma série de vantagens quando comparada com o modelo tradicional sequencial, isto é o reator seguido de coluna de destilação [2]. Sabe-se que as colunas de destilação são responsáveis por grande parte do consumo energético das plantas de processamento [3], além disso, as unidades destiladoras convencionais apresentam baixa eficiência energética [4]. Desta maneira, o presente trabalho busca desenvolver estudos do comportamento termoquímico suportado na ferramenta computacional Aspen Plusâ, com o intuito de entender a termoquímica e comportamento dos componentes envolvidos, assim como a sua termoquímica, interação das misturas de equilibro e reações na produção do
TAME. Também, busca-se avaliar o comportamento fenomenológico do processo.

\section{Caraterização termoquímica e termodinâmica}

A caraterização da termodinâmica e termoquímica são de suma importância para o entendimento dos fenômenos associados ao equilibro e da energia disponível. Além do comportamento energético, dos balanços e das relações entre as leis da termodinâmica, a termoquímica nos fornece a relação de equilíbro entre as fases líquida e vapor. O equilibro do processo descreve a interação e comportamento dos componentes da mistura com o intuito de obter TAME em uma unidade intensificada de separação e reação química. Estudos das unidades com aproveitamento energético têm sido estudados em pesquisas ao longo do mundo $[5,6]$. Visando uma melhor compreensão da termoquímica, duas abordagens têm sido apresentadas: a primeira, uma equação de estado para modelar as fases líquida e vapor com o auxílio de coeficientes de atividade e a segunda, uma equação de estado para modelar ambas fases. Assim, parâmetros binários para o método UNIFAC foram usados para o cálculo do equilíbrio na produção do TAME, sendo que este encontra-se disponível na base de dados do simulador[7].

O estudo possibilita a obtenção do TAME na configuração de coluna reativa - RD (constituída por zona reativa, holdup, número de pratos, pressão, temperatura, refervedor e condensador) e configuração encontrada na literatura aberta [8]. 
No processo de produção do TAME e, estimação de parâmetros considera-se que a reação acontece na fase líquida para o equilíbrio de conversão. A reação é descrita no simulador na forma cinética power-low no estado estacionário.

\section{Método UNIFAC}

UNIFAC é um método de coeficientes de atividade, está suportado em contribuição de grupos, especificamente das contribuições moleculares. Este modelo é limitado pelo número de parâmetros e a interação entre os grupos. O modelo original do UNIFAC permite a caraterização do equilíbrio líquido-vapor (VLE) e do equilíbrio líquido-liquido (LLE).

A equação do modelo base UNIFAC descreve termos combinatórios e residuais, apresentado a seguir [9]:

$$
\begin{gathered}
\ln \gamma=\ln \gamma_{i}^{C}+\ln \gamma_{i}^{r} \\
\ln \gamma_{\mathrm{i}}^{C}=\ln \left(\frac{\Phi_{\mathrm{i}}}{\mathrm{x}_{\mathrm{i}}}\right)+1-\frac{\Phi_{\mathrm{i}}}{\mathrm{x}_{\mathrm{i}}}-\frac{\mathrm{Z}}{2}
\end{gathered}
$$

Onde as frações de superfície e volume molecular são:

$$
\Phi_{i}=\frac{x_{i} r_{i}}{\sum_{j}^{n c} x_{j} r_{j}} \quad \text { e } \quad \theta_{i}=\frac{x_{i} \frac{z}{2} q_{i}}{\sum_{j}^{n c} x_{j} \frac{z}{2} q_{j}}
$$

Assim, $n c$ é o número de componentes da mistura. O número de coordenação $z$ é fixado em 10 de forma estandard. Os parâmetros $r_{i}$ e $q_{i}$ são calculados desde o grupo de volume e parâmetros de área, segundo a equação:

$$
\mathrm{r}_{\mathrm{i}}=\sum_{\mathrm{k}}^{\mathrm{ng}} \mathrm{v}_{\mathrm{ki}} \mathrm{R}_{\mathrm{k}} \text { e } \quad \mathrm{q}_{\mathrm{i}}=\sum_{\mathrm{k}}^{\mathrm{ng}} \mathrm{v}_{\mathrm{ki}} \mathrm{Q}_{\mathrm{k}}
$$

Onde $v_{k i}$ é o número de grupos tipo $k$ em moléculas $i$, e $n g$ é o número de grupos na mistura. Os termos residuais são descritos como:

$$
\ln \gamma_{\mathrm{i}}^{\mathrm{r}}=\sum_{\mathrm{k}}^{\mathrm{ng}} \mathrm{v}_{\mathrm{ki}}\left[\ln \Gamma_{\mathrm{k}}-\ln \Gamma_{\mathrm{k}}^{\mathrm{i}}\right]
$$

$\Gamma_{k}$ é o coeficiente de atividade de um grupo a uma composição de mistura, e $\Gamma_{k}{ }^{i}$ é o coeficiente de atividade do grupo $k$ na mistura do grupo correspondente ao puro $i$. Os parâmetros $\Gamma_{k}$ e $\Gamma_{k}^{i}$ são definidos como:

$$
\ln \Gamma_{\mathrm{k}}=\mathrm{Q}_{\mathrm{k}}\left[1-\ln \sum_{\mathrm{m}}^{\mathrm{ng}} \theta_{\mathrm{m}} \tau_{\mathrm{mk}}-\sum_{\mathrm{m}}^{\mathrm{ng}}\left(\frac{\theta_{\mathrm{m}} \tau_{\mathrm{km}}}{\sum_{\mathrm{n}}^{\mathrm{ng}} \theta_{\mathrm{n}} \tau_{\mathrm{nm}}}\right)\right]
$$

Quando:

$$
\begin{gathered}
\theta_{\mathrm{k}}=\frac{\mathrm{x}_{\mathrm{k}} \frac{\mathrm{z}}{2} Q_{\mathrm{k}}}{\sum_{\mathrm{m}}^{\mathrm{ng}} \mathrm{x}_{\mathrm{m}} \frac{\mathrm{z}}{2} Q_{\mathrm{m}}} \\
\tau_{\mathrm{nm}}=\mathrm{e}^{-\mathrm{b}_{\mathrm{mn}} / \mathrm{T}}
\end{gathered}
$$

Onde o parâmetro $X_{k}$ é o grupo de fração molar do grupo $k$ no líquido:

$$
\mathrm{X}_{\mathrm{k}}=\frac{\sum_{\mathrm{j}}^{\mathrm{nc}} \mathrm{v}_{\mathrm{kj}} \mathrm{x}_{\mathrm{j}}}{\sum_{\mathrm{j}}^{\mathrm{nc}} \sum_{\mathrm{m}}^{\mathrm{ng}} \mathrm{v}_{\mathrm{mj}} \mathrm{X}_{\mathrm{j}}}
$$

Sendo $k, m, v_{m}, v_{k}$ o número de ocorrências em cada grupo.

\section{Considerações gerais}

Sabe-se que a representação de um sistema por meio de um modelo é fortemente dependente das informações de entrada, por tanto, um amplo número de dados e propriedades termodinâmicas são absolutamente necessários para simular o processo. O simulador de processos Aspen Plus V9® contém as informações necessárias para a simulação do sistema para a produção do TAME.

\section{Fatores Considerados}

O sistema de estudo envolve os seguintes compostos: 2M1B (2-metil, 1-buteno), 2M2B (2-metil, 2-buteno), TAME (metil-ter-pentil-eter), NC5 (n-pentano), IC5(2-metil butano), 1 Pentano e 2 Pentano para o sistema de síntese do TAME.

As qualidades dos produtos encontram-se relacionadas com aspectos operacionais e descrição matemática, sendo assim, as variáveis fundamentais do processo na unidade intensificada são as que determinam as condições de operação. Os processos dependem das variáveis:

- Calor da reação do processo.

- Composição do óleo.

- Pressão da coluna.

- Carga térmica requerida pelo refervedor e condensador.

- Vazão de refluxo da coluna de destilação.

- Número de pratos

A análise termodinâmica determina o déficit da entalpia líquida e as perdas exegéticas devido à irreversibilidade (resultado da entropia). Assim, o estudo da unidade de processo, desde a ótica 
dos pontos onde não se apresenta distribuição uniforme, estão sendo foco de melhorias. A redução da irreversibilidade permite o aumento do trabalho potencial disponível na unidade de processo, da mesma forma, uma diminuição do consumo energético e aumento da eficiência, suportado no conceito de um estado termodinâmico mínimo (ETM).

Os fatores a serem considerados no desenvolvimento do sistema intensificado de destilação e reação química são:

- Estágio de alimentação: permite minimizar desvios significativos da entalpia no estágio relativo ao local de alimentação.

- Alterações na vazão de refluxo: uma menor vazão de refluxo reduz o consumo energético de uma coluna convencional.

- Mudança da temperatura de alimentação: promoção de perdas energéticas semelhantes tanto no condensador e no refervedor.

No caso do TAME, a proposta do sistema intensificado tem como requisito básico manter - grau de pureza no sistema convencional e assim, observar o consumo energético na unidade de processo. Por tanto, o esquema de análise termodinâmico é desenvolvido de forma sequencial como é apresentado na Figura 1 em relação á configuração de destilação reativa apresentada por León 2017 [10].

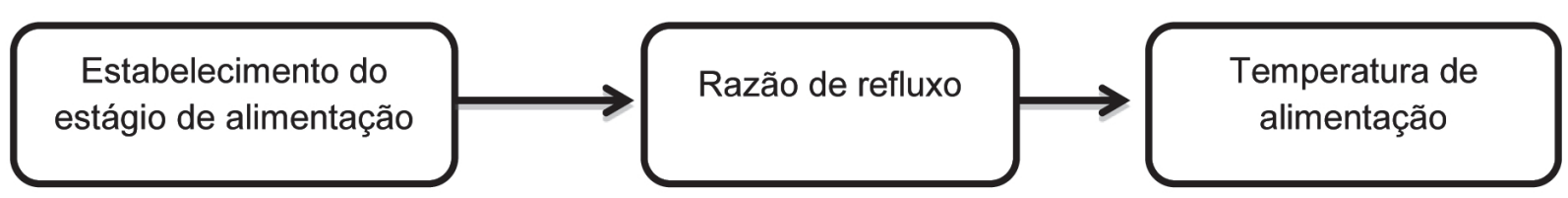

Figura 1. Estrutura sequencial da caraterização no simulador para a RD.

\section{Metodologia}

A metodologia desenvolvida para o sistema de destilação adotou uma configuração de processo onde foi simulada a obtenção do TAME. O arranjo de coluna a ser aplicado, inclui uma configuração interna para o aproveitamento da energia, o qual gera as maiores causas de divergências no sistema. O primeiro passodefine informações dos componentes puros, suas propriedades físicas e químicas, dados fundamentais para o estudo do equilíbrio, e o segundo pela convergência da reação (estimações para o desenvolvimento e cálculo da reação). Consequentemente, o terceiro procura a convergência do estudo das misturas binárias fazendo uso do método termodinâmico. De modo a garantir a convergência do sistema, a simulação é analisada.

\section{Parâmetros e estudo dos componentes}

As simulações foram desenvolvidas no simulador de processos Aspen Plus V9®, sendo uma ferramenta amplamente usada na indústria química.. No caso do TAME, no simulador encontram-se os componentes necessários para a síntese. A síntese do TAME envolve uma reação exotérmica, em consequência, propriedades físicas e químicas permitem estimar os parâmetros no equilíbrio na obtenção deste aditivo da gasolina, um resumo das propriedades é apresentado na Tabela 1 a seguir.

Os componentes que participam no processo de obtenção do TAME são o Penteno (1Pentene) e Trans-2-Penteno (2Pentene), o metanol (MEOH), o Pentano (NC5), Butano Metílico (IC5), Buteno 1-metilico (2M1B), Buteno 2-metilico (2M2B) e $(\mathrm{H} 2 \mathrm{O})$ de acordo com o presentado na Tabela 1. A pressão de vapor descreve o comportamento da pressão da fase vapor sobre a fase líquida, o equilíbro dinâmico acontece a uma temperatura determinada, em consequência, o comportamento é indiferente da quantidade de massa. O estudo dos componentes e da pressão de vapor experimentada às diferentes temperaturas são apresentadas na Figura 2.

O comportamento da pressão de vapor para os componentes puros apresenta um incremento relativamente constante no intervalo de temperatura entre $280 \mathrm{~K}\left(6,8^{\circ} \mathrm{C}\right)$ e $360 \mathrm{~K}\left(86,85^{\circ} \mathrm{C}\right)$. Os produtos obtidos da reação apresentam maior pressão de vapor no intervalo de temperatura estudado (IC5, 2M1B, 2M2B), no entanto, o produto de interesse TAME apresenta uma pressão de vapor menor, favorecendo a estabilidade no comportamento, no intervalo de temperatura estudado. 
rev.ion. 2019;32(1):87-95. Bucaramanga (Colombia).

Tabela 1. Propriedades dos Componentes - Síntese TAME.

\begin{tabular}{|c|c|c|c|c|c|c|c|c|c|}
\hline Propriedade/Unidades & MEOH & 1-PENTEN & 2-PENTEN & NC5 & IC5 & 2M1B & 2M2B & TAME & $\mathrm{H} 2 \mathrm{O}$ \\
\hline $\begin{array}{l}\text { Gibbs Padrão de } \\
\text { Formação (J/kmol) }\end{array}$ & $-1,62 \mathrm{E}+8$ & $7,84 \mathrm{E}+7$ & $6,98 \mathrm{E}+7$ & $-8,81 \mathrm{E}+6$ & $-1,41 \mathrm{E}+7$ & $6,67 \mathrm{E}+7$ & $6,05 \mathrm{E}+7$ & $-1,14 \mathrm{E}+8$ & $-2,29 \mathrm{E}+8$ \\
\hline $\begin{array}{l}\text { Entalpia Padrão de } \\
\text { Formação (J/kmol) }\end{array}$ & $-2,01 \mathrm{E}+8$ & $-2,16 \mathrm{E}+7$ & $-3,11 \mathrm{E}+7$ & $-1,47 E+8$ & $-1,54 \mathrm{E}+8$ & $-3,53 \mathrm{E}+7$ & $-4,18 \mathrm{E}+7$ & $-3,05 E+8$ & $-2,42 \mathrm{E}+8$ \\
\hline $\begin{array}{c}\text { Entalpia de } \\
\text { Vaporização (J/kmol) }\end{array}$ & $3,51 \mathrm{E}+7$ & $2,51 \mathrm{E}+7$ & $2,63 \mathrm{E}+7$ & $2,58 \mathrm{E}+7$ & $2,48 \mathrm{E}+7$ & $2,56 \mathrm{E}+7$ & $2,65 \mathrm{E}+7$ & $3,07 \mathrm{E}+7$ & $4,08 \mathrm{E}+7$ \\
\hline $\begin{array}{l}\text { Entalpia padrão de } \\
\text { combustão }(\mathrm{J} / \mathrm{kmol})\end{array}$ & $-6,38 \mathrm{E}+8$ & $-3,13 E+9$ & $-3,12 E+9$ & $-3,24 \mathrm{E}+9$ & $-3,24 \mathrm{E}+9$ & $-3,12 \mathrm{E}+9$ & $-3,11 \mathrm{E}+9$ & $-3,71 \mathrm{E}+9$ & - \\
\hline Massa Molecular & 32,0422 & 70,1344 & 70,1344 & 72,1503 & 72,1503 & 70,1344 & 70,1344 & 102,177 & 18,0153 \\
\hline $\begin{array}{l}\text { Pressão crítica (N/ } \\
\left.\qquad m^{\wedge} 2\right)\end{array}$ & $8,08 \mathrm{E}+6$ & $3,56 \mathrm{E}+6$ & $3,66 \mathrm{E}+6$ & $3,37 \mathrm{E}+6$ & $3,38 \mathrm{E}+6$ & $3,45 \mathrm{E}+6$ & $3,42 \mathrm{E}+6$ & $3,04 \mathrm{E}+6$ & $2,21 \mathrm{E}+7$ \\
\hline $\begin{array}{c}\text { Gravidade Específica } \\
\text { Padrão }\end{array}$ & 0,7962 & 0,6457 & 0,6533 & 0,631 & 0,6247 & 0,6557 & 0,6676 & 0,76765 & 1 \\
\hline $\begin{array}{l}\text { Temperatura de } \\
\text { Ebulição (K) }\end{array}$ & 337,85 & 303,22 & 309,49 & 309,22 & 300,994 & 304,305 & 311,705 & 359,51 & 373,15 \\
\hline Temperatura crítica (K) & 512,5 & 464,8 & 474,2 & 469,7 & 460,4 & 465 & 470 & 534 & 647,096 \\
\hline VB (cum/kmol) & 0,04274 & 0,11130 & 0,111122 & 0,11819 & 0,11762 & 0,10985 & 0,10946 & 0,14501 & 0,018831 \\
\hline $\begin{array}{l}\text { Volume crítico (cum/ } \\
\text { kmol) }\end{array}$ & 0,117 & 0,2934 & 0,293 & 0,313 & 0,306 & 0,292 & 0,292 & 0,386 & 0,0559472 \\
\hline $\begin{array}{c}\text { Fator de } \\
\text { Compressibilidade } \\
\text { Crítica }\end{array}$ & 0,222 & 0,27 & 0,272 & 0,27 & 0,27 & 0,26 & 0,256 & 0,264 & 0,229 \\
\hline
\end{tabular}

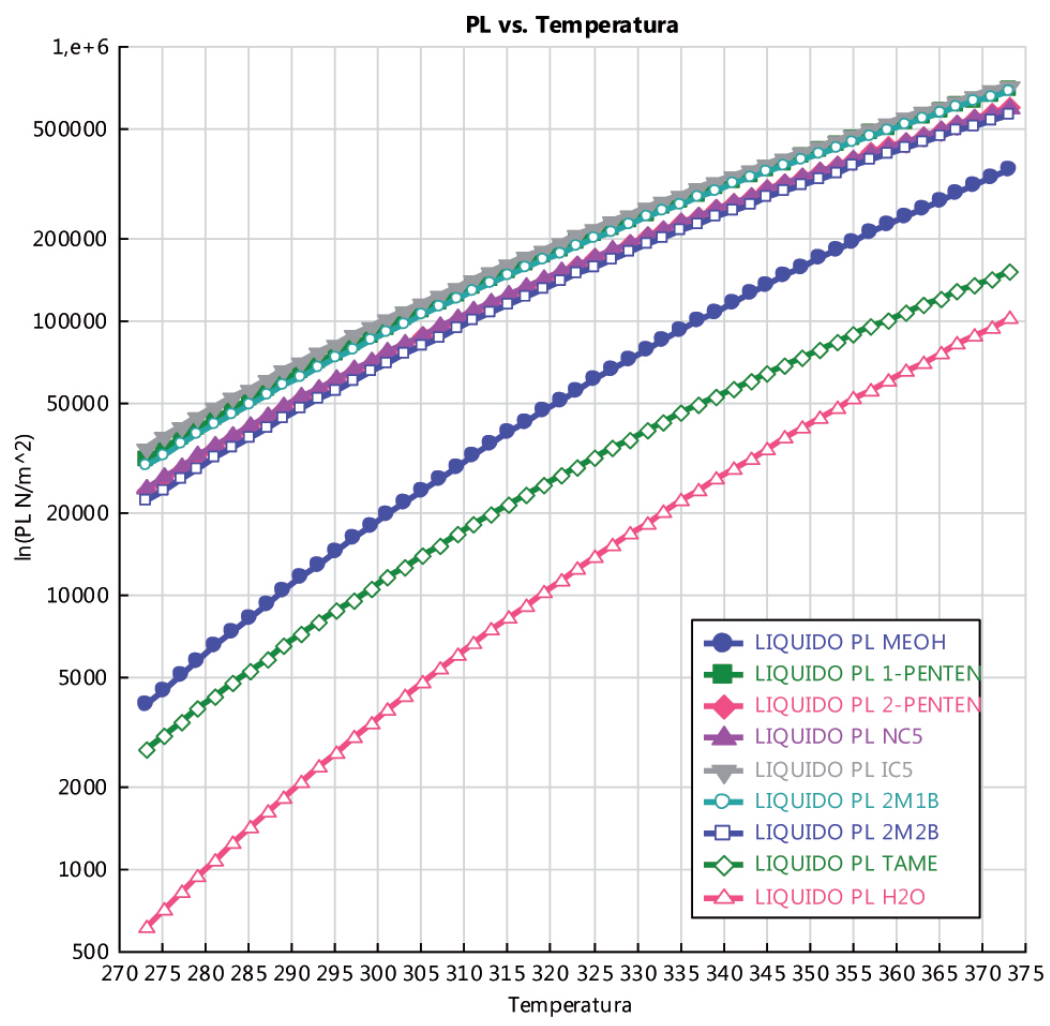

Figura 2. Diagrama Pressão de Vapor vérsus Temperatura (K) dos Componentes de Síntese do TAME. 
Determinação de Parâmetros do Equilíbrio Binário dos Componentes

Os estudos dos componentes foram avaliados em relação aos modelos termodinâmicos UNIFAC, UNIQUAC, NRTL, PENG ROBINSON. Uma vez estudadas as propriedades dos componentes e as fases presentes na operação, o método UNIFAC representa de forma ajustada os componentes para a síntese do TAME. Estes além de ser sugeridos na literatura são os que apresentam maior faixa de aplicabilidade para os componentes de estudo, dadas as condições de operação. O método curto de análise "Property method selection" do simulador Aspen Plus V9® foi usado com o objetivo de ter uma validação previa dos modelos possíveis disponíveis no simulador, sendo escolhido o método UNIFAC.

O método UNIFAC, usa a contribuição molecular e uma interação de parâmetros grupo a grupo para a predição, e assim, o cálculo dos coeficientes de atividade. Os métodos têm a restrição de temperatura mínima de $290 \mathrm{~K}$ e máxima de $420 \mathrm{~K}$, sendo estimado o equilíbrio Líquido-Vapor (LVE) o Líquido-Líquido (LLE) nesta faixa de temperatura.

\section{Parâmetros de Equilíbrio da Síntese do TAME}

Os componentes envolvidos na produção do TAME foram avaliados com o objetivo de conhecer a fenomenologia do processo, assim, uma análise dos componentes e seu comportamento no equilíbrio de fases permitiu conhecer possíveis azeótropos e comportamentos particulares dos componentes em mistura.

Como pode-se perceber, o sistema é de grande complexidade para a simulação, os parâmetros foram estimados por meio do simulador. Os parâmetros de área do modelo de atividade UNIFAC (GMUFQ) e parâmetros de volume (GMUFR) são apresentados na Tabela 2.

Os parâmetros do grupo binário estimados para o modelo UNIFAC (GMUFB) são apresentados, a seguir na Tabela 3.

Tabela 2. Parâmetros de Área e Volume Modelo UNIFAC - Síntese TAME.

\begin{tabular}{ccccccccccc}
\hline Parâmetros & $\begin{array}{c}\text { Grupo ID } \\
\mathbf{1 2 5 0}\end{array}$ & $\begin{array}{c}\text { Grupo } \\
\text { ID 1010 }\end{array}$ & $\begin{array}{c}\text { Grupo } \\
\text { ID 1015 }\end{array}$ & $\begin{array}{c}\text { Grupo } \\
\text { ID 1060 }\end{array}$ & $\begin{array}{c}\text { Grupo } \\
\text { ID 1055 }\end{array}$ & $\begin{array}{c}\text { Grupo } \\
\text { ID 1000 }\end{array}$ & $\begin{array}{c}\text { Grupo } \\
\text { ID 1615 }\end{array}$ & $\begin{array}{c}\text { Grupo } \\
\text { ID 1005 }\end{array}$ & $\begin{array}{c}\text { Grupo } \\
\text { ID 1070 }\end{array}$ & $\begin{array}{c}\text { Grupo } \\
\text { ID 1065 }\end{array}$ \\
\hline GMUFQ & 1,432 & 0,54 & 0,848 & 0,988 & 0,676 & 0 & 1,088 & 0,228 & 1,176 & 0,867 \\
GMUFR & 1,4311 & 0,674 & 0,9011 & 1,1173 & 0,8886 & 0,2195 & 1,145 & 0,4469 & 1,3454 & 1,1167 \\
\hline
\end{tabular}

Tabela 3. Parâmetros binários Modelo UNIFAC - Síntese TAME.

\begin{tabular}{cccccccccccc}
\hline Grupo i/j - ID & 1250 & 1010 & 1015 & 1060 & 1055 & 1000 & 1615 & 1005 & 1070 & 1065 \\
\hline 1250 & 0 & 16,51 & 16,51 & $-12,52$ & $-12,52$ & 16,51 & $-128,6$ & 16,51 & $-12,52$ & $-12,52$ \\
1010 & 697,2 & 0 & 0 & 86,02 & 86,02 & 0 & 251,5 & 0 & 86,02 & 86,02 \\
1015 & 697,2 & 0 & 0 & 86,02 & 86,02 & 0 & 251,5 & 0 & 86,02 & 86,02 \\
1060 & 787,6 & $-35,36$ & $-35,36$ & 0 & 0 & $-35,36$ & 214,5 & $-35,36$ & 0 & 0 \\
1055 & 787,6 & $-35,36$ & $-35,36$ & 0 & 0 & $-35,36$ & 214,5 & $-35,36$ & 0 & 0 \\
1000 & 697,2 & 0 & 0 & 86,02 & 86,02 & 0 & 251,5 & 0 & 86,02 & 86,02 \\
1615 & 238,4 & 83,36 & 83,36 & 26,51 & 26,51 & 83,36 & 0 & 83,36 & 26,51 & 26,51 \\
1005 & 697,2 & 0 & 0 & 86,02 & 86,02 & 0 & 251,5 & 0 & 86,02 & 86,02 \\
1070 & 787,6 & $-35,36$ & $-35,36$ & 0 & 0 & $-35,36$ & 214,5 & $-35,36$ & 0 & 0 \\
1065 & 787,6 & $-35,36$ & $-35,36$ & 0 & 0 & $-35,36$ & 214,5 & $-35,36$ & 0 & 0
\end{tabular}


$\mathrm{Na}$ síntese do TAME o metanol apresenta um comportamento azeotrópico em mistura, levando em consideração que a configuração adotada apresenta duas seções da coluna que operam a pressões diferentes, a possibilidade de obter 0 metanol puro esta diretamente ligado às condições de operação.

Na seção de esgotamento foi estabelecida uma operação convencional de 4 bar. e para a seção de retificação uma pressão de 10 bar [11]. Esta diferença de pressão força a mudança do ponto azeotrópico da mistura, a qual é desfavorecida pelo aumento da pressão, indicando que o metanol na fase vapor é arrastrado para baixas temperaturas. Isto dificulta a separação deste componente, o que pode ser observado nas Figura 3.

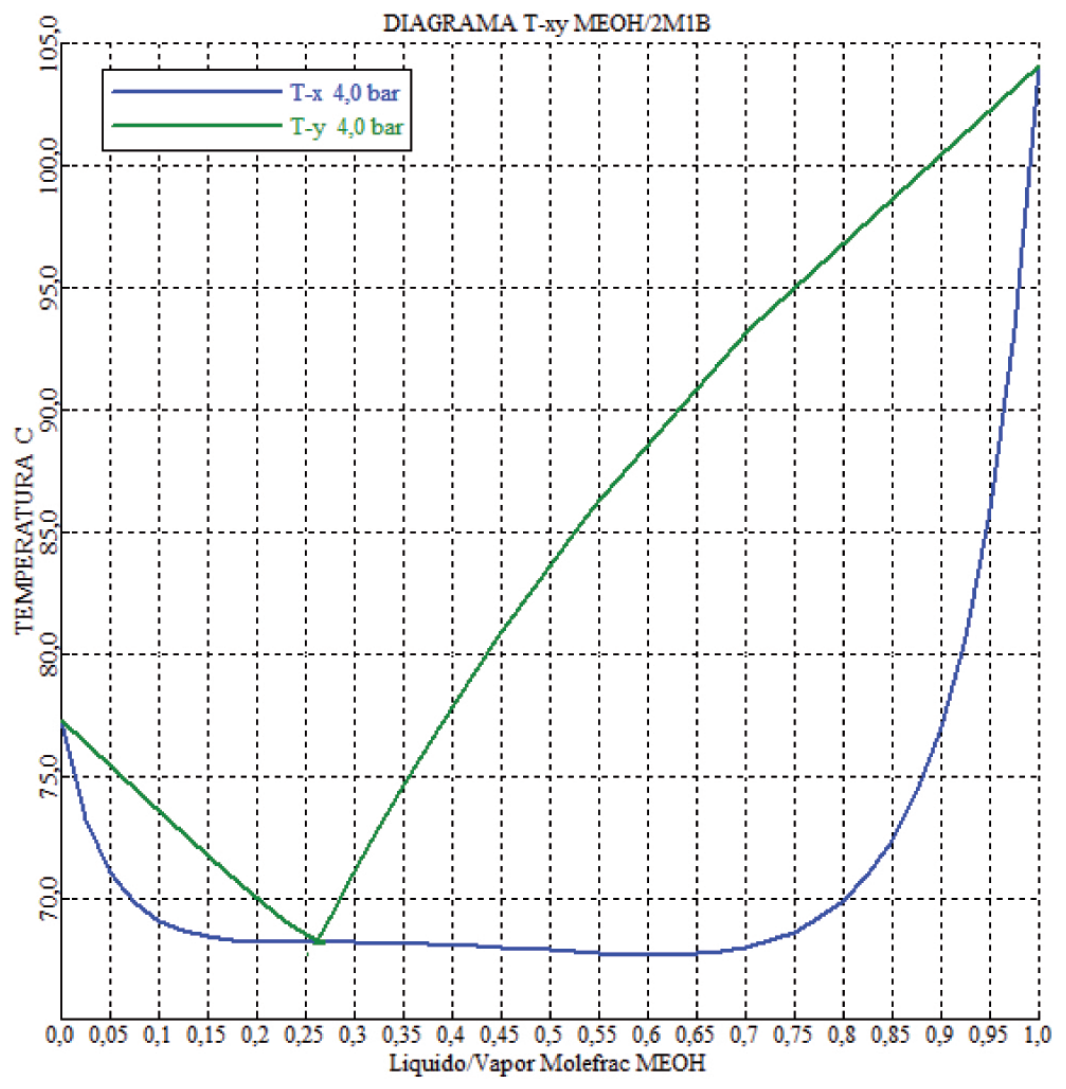

Figura 3. Diagrama T-xy Metanol-2M1B a 4 bar (4,08 atm).

O azeótropo evidenciado faz referência ao comportamento da relação e composição química, outros pontos são encontrados na pressão padrão a diferentes temperaturas. Assim, a mistura apresenta nove azeótropos nas fases líquida e vapor. As concentrações atingidas a diferentes temperaturas para os pares binários que interagem no processo são apresentadas na Tabela 4. Pode ser observado que estes aparecem entre as temperaturas de $64,4{ }^{\circ} \mathrm{C}$ e $86,28{ }^{\circ} \mathrm{C}$. Na Tabela 5 são apresentadas as bases molar e mássica dos componentes descritos em pares binários nas temperaturas de análise azeotrópico.
Tabela 4. Estudo da Temperatura Azeotrópica da Mistura a 1 ATM - Síntese TAME.

\begin{tabular}{ccc}
\hline Componente & Clasificação & Temperatura \\
\hline MEOH & Nodo estável & $148,07^{\circ} \mathrm{F}\left(64,4^{\circ} \mathrm{C}\right)$ \\
2M1B & Saddle & $88,08^{\circ} \mathrm{F}\left(27,7^{\circ} \mathrm{C}\right)$ \\
2M2B & Saddle & $101,30^{\circ} \mathrm{F}\left(38,5^{\circ} \mathrm{C}\right)$ \\
TAME & Nodo estável & $187,30^{\circ} \mathrm{F}\left(86,28^{\circ} \mathrm{C}\right)$ \\
NC5 & Saddle & $96,88^{\circ} \mathrm{F}\left(36,04^{\circ} \mathrm{C}\right)$ \\
IC5 & Saddle & $82,14^{\circ} \mathrm{F}$ \\
1-PENTE & Saddle & $86,10^{\circ} \mathrm{F}$ \\
2-PENTE & Saddle & $98,49^{\circ} \mathrm{F}$ \\
\hline
\end{tabular}


rev.ion. 2019;32(1):87-95. Bucaramanga (Colombia).

Tabela 5. Estudo Azeotrópico da Mistura a 1 ATM - Síntese TAME.

\begin{tabular}{|c|c|c|}
\hline Par binario & \multicolumn{2}{|c|}{ Temperatura $81,15^{\circ} \mathrm{F}\left(29,53^{\circ} \mathrm{C}\right)$} \\
\hline & Base Molar & Base Mássica \\
\hline MEOH & 0,1826 & 0,0926 \\
\hline 2M1B & 0,8174 & 0,9074 \\
\hline Par binario & \multicolumn{2}{|c|}{ Temperatura $90.76^{\circ} \mathrm{F}\left(32.64^{\circ} \mathrm{C}\right)$} \\
\hline MEOH & 0,2376 & 0,1246 \\
\hline 2M2B & 0,7624 & 0,8754 \\
\hline Par binario & \multicolumn{2}{|c|}{ Temperatura $145.01^{\circ} \mathrm{F}\left(62.78^{\circ} \mathrm{C}\right)$} \\
\hline MEOH & 0,7861 & 0,5355 \\
\hline TAME & 0,2139 & 0,4645 \\
\hline Par binario & \multicolumn{2}{|c|}{ Temperatura $87.11^{\circ} \mathrm{F}\left(30.62^{\circ} \mathrm{C}\right)$} \\
\hline MEOH & 0,2296 & 0,1169 \\
\hline NC5 & 0,7704 & 0,8831 \\
\hline Par binario & \multicolumn{2}{|c|}{ Temperatura $76.11^{\circ} \mathrm{F}\left(24.51^{\circ} \mathrm{C}\right)$} \\
\hline MEOH & 0,1705 & 0,0836 \\
\hline IC5 & 0,8295 & 0,9164 \\
\hline Par binario & \multicolumn{2}{|c|}{ Temperatura $79.52^{\circ} \mathrm{F}\left(26.4^{\circ} \mathrm{C}\right)$} \\
\hline MEOH & 0,1728 & 0,0871 \\
\hline 1-PENTE & 0,8272 & 0,9129 \\
\hline Par binario & \multicolumn{2}{|c|}{ Temperatura $88.85^{\circ} \mathrm{F}\left(31.58^{\circ} \mathrm{C}\right)$} \\
\hline MEOH & 0,223 & 0,1159 \\
\hline 2-PENTE & 0,777 & 0,8841 \\
\hline Par binario & \multicolumn{2}{|c|}{ Temperatura $96.84^{\circ} \mathrm{F}\left(36.02^{\circ} \mathrm{C}\right)$} \\
\hline NC5 & 0,854 & 0,8575 \\
\hline 2-PENTE & 0,146 & 0,1425 \\
\hline Par binario & \multicolumn{2}{|c|}{ Temperatura $82.13^{\circ} \mathrm{F}\left(27.85^{\circ} \mathrm{C}\right)$} \\
\hline IC5 & 0,9682 & 0,9691 \\
\hline 1-PENTE & 0,0318 & 0,0309 \\
\hline
\end{tabular}

O diagrama ternário da mistura é obtido das temperaturas de mudança de fase dos pelo simulador (Figura 4), apresentando o componentes envolvidos na obtenção do TAME. comportamento das concentrações dentro

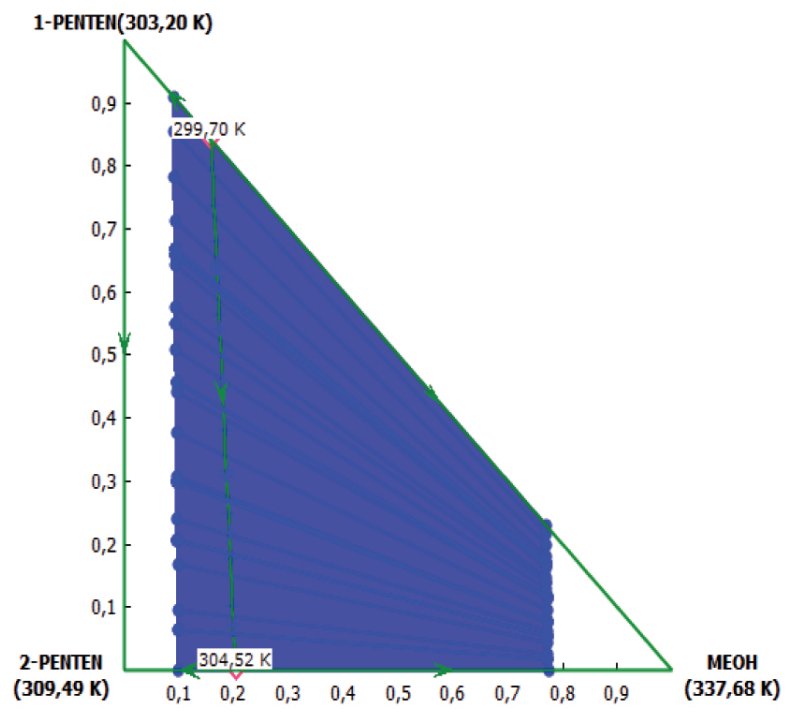

Figura 4. Diagrama Ternario1-Pentene, 2-Pentene e MEOH. 
No diagrama é observado que entre as temperaturas de $299,7 \mathrm{~K}\left(26,55^{\circ} \mathrm{C}\right)$ e $304,52 \mathrm{~K}$ $\left(31,37^{\circ} \mathrm{C}\right)$ encontram-se nós de temperaturas instáveis pela presença do azeótropo atingido às concentrações mássicas de 0,1 e 0,07 do MEOH.

\section{Conclusões}

As particularidades dos sistemas e o comportamento azeotrópico das misturas foram identificadas no simulador de processos Aspen Plus V9 lebando em conta. o estudo dos fenómenos e das dificuldades da mistura de componentes na produção do TAME. A análise dos modelos termodinâmicos apresentam a presença de um azeótropo, isto dificulta o processo de destilação. Os processos de destilação azeotrópica apresentam grandes desafios, sendo um processo mais complexo que o convencional, isto porque a convergência e o cálculo do equilíbrio não é simples devido à necessidade de estimação de dados e à parametrização de componentes. Por outro lado, o processo apresentou-se bastante sensível a variações tornando ainda mais complexa a simulação. O método UNIFAC permite estimar o equilíbrio com precisão para o sistema de estudo TAME descrevendo nove pontos azeotrópicos a diferentes temperaturas para os pares binários na faixa de $(24,51-62,78)^{\circ} \mathrm{C}$. As pressões de vapor são fundamentais para o equilíbro de fases, sendo que a condição do processo está em função dos pontos críticos dos componentes e da temperatura onde se apresentam os azeótropos $(6,8-86,85)$ ${ }^{\circ} \mathrm{C}$. O diagrama ternário desenvolvido descreve o comportamento das concentrações em relação às misturas em condições de pressão constante.

\section{Agradecimentos}

Os autores agradecem à Universidad EAN, à Universidad de Cartagena, à Universidad Industrial de Santander, à Universidade Federal de São Paulo e à Universidade Estadual de Campinas pelo apoio.

\section{Referências}

[1] Al-Arfaj MA, Luyben W. Plantwide control for TAME production using reactive distillation. AIChE J. 2004;50:1462-73.

[2] Tuchlenski A, Beckmann A, Reusch D, Düssel R, Weidlich $U$, Janowsky R. Reactive distillation-industrial applications, process design \& scale-up. Chem. Eng. Sci. 2001;56:387-94.

[3] Vanaki AS, Eslamloueyan R. Steady-state simulation of a reactive internally heat integrated distillation column (R-HIDiC) for synthesis of tertiary-amyl methyl ether (TAME). Chem. Eng. Process. 2012;52:21-7.

[4] Nakaiwa M, Huang K, Endo A, Ohmori T,Akiya T, Takamatsu T. Internally Heat integrated distillation columns: A review. Chem. Eng. Res. Des. 2003;81:162-77.

[5] León Pulido J, Martinez EL, Bineli ARR; Wolf MR, Filho RM. Heat Transfer Study in a Concentric Stage of an Internally HeatIntegrated Distillation Column (HIDiC) Using CFD Simulation. In:AoSI, Douglas C, Grundfest WS, Burgstone J, editors. Proceedings of the World Congress on Engineering and Computer Science. 2010 oct 20-22; San Francisco, USA. San Francisco: Newswood Limited; 2010. p. 742-7.

[6] León Pulido J, Wolf MR, Filho RM. Nuevas Perspectivas en Procesos de Separación: Simulación Columna de Destilación con Integración Interna de Calor (CDIIC). rev.ion 2010;23(1):7-12.

[7] Levenspiel $O$. Unsteady-state heating and cooling of solid objects. In: Engineering flow and Heat Exchanger. Levenspiel O, Editor. USA: Springer; 1993. p. 245-59.

[8] Luyben LW. Comparison of Pressure-Swing and Extractive-Distillation Methods for Methanol-Recovery Systems in the TAME Reactive-Distillation Process. Ind. Eng. Chem. Res. 2005;44(15):5715-25

[9] Hansen HK, Rasmussen P, Fredenslund A, Schiller M, Gmehling J. Vapor-liquid equilibria by UNIFAC group contribution. 5 . Revision and extension. Ind. Eng. Chem. Res. 1991;30(10):2352-5.

[10]Leon Pulido J, Sarmiento MP, Garzon DM, Hernandez MA, Gonzalez AD, Peralta Ruiz YY, Duran M. Energy Study of Reactive-HIDiC Simulation for Ethyl Acetate Synthesis from Acetic Acid. Chem. Eng. Trans. 2017;58:54752.

[11] León Pulido J. Estudo de um novo conceito de coluna de destilação: coluna de destilação com integração interna de calor (CDIIC) (Dissertação de Mestrado). São Paulo, Brasil: Universidade Estadual de Campinas; 2011. 